\title{
Transition region, coronal heating and the fast solar wind
}

\begin{abstract}
Xing $\mathrm{Li}^{\star}$
Department of Physics, University of Wales, Aberystwyth, SY23 3BZ, UK

Received 31 October 2002 / Accepted 28 April 2003

Abstract. It is assumed that magnetic flux tubes are strongly concentrated at the boundaries of supergranule convection cells. A power law spectrum of high frequency Alfvén waves with a spectral index -1 originating from the sun is assumed to supply all the energy needed to energize the plasma flowing in such magnetic flux tubes. At the high frequency end, the waves are eroded by ions due to ion cyclotron resonance. The magnetic flux concentration is essential since it allows a sufficiently strong energy flux to be carried by high frequency ion cyclotron waves and these waves can be readily released at the coronal base by cyclotron resonance. The main results are: 1 . The waves are capable of creating a steep transition region, a hot corona and a fast solar wind if both the wave frequency is high enough and the magnetic flux concentration is sufficiently strong in the boundaries of the supergranule convection zone. 2. By primarily heating alpha particles only, it is possible to produce a steep transition region, a hot corona and a fast solar wind. Coulomb coupling plays a key role in transferring the thermal energy of alpha particles to protons and electrons at the corona base. The electron thermal conduction then does the remaining job to create a sharp transition region. 3. Plasma species (even ions) may already partially lose thermal equilibrium in the transition region, and minor ions may already be faster than protons at the very base of the corona. 4 . The model predicts high temperature alpha particles $\left(T_{\alpha} \sim 2 \times 10^{7} \mathrm{~K}\right)$ and low proton temperatures $\left(T_{\mathrm{p}}<10^{6} \mathrm{~K}\right)$ between 2 and 4 solar radii, suggesting that hydrogen Lyman lines observed by UVCS above coronal holes may be primarily broadened by Alfvén waves in this range.
\end{abstract}

Key words. Sun: corona - Sun: solar wind - Sun: transition region

\section{Introduction}

It has been well known for several decades that the solar wind is the expansion of the hot corona along open magnetic field flux tubes. However, the exact mechanisms responsible for the creation of a hot corona and the acceleration of the fast solar wind continue to be an unresolved issue (Axford et al. 1999; Marsch 1999). Since the fast solar wind streams and the corona are connected, there has been a growing awareness that the two problems should be treated in a self-consistent way. It is also natural to seek a single theory to account for the coronal heating and the acceleration of the fast solar wind along open magnetic field fluxes. However, the Sun and the inner corona are far away, the environment near the Sun is harsh for the survival of space probes, so in situ measurements of the plasma parameters in the near Sun region are still not available. Without in situ measurements, it is difficult to tell what is responsible for the coronal heating and the acceleration of the fast solar wind. However, as the fast solar wind cruises into interplanetary space, manmade satellites have been able to detect the properties of the fast (and slow) solar wind streams over the past few decades. These direct measurements have shown that Alfvén waves are ubiquitous in fast solar wind streams (e.g., Belcher \& Davis 1971; Smith et al. 1995). They provide an attractive mechanism for the solar wind heating and acceleration if their high

^ e-mail: xxl@aber.ac.uk frequency counterparts, whose energy can be directly absorbed by the solar wind plasmas, exist in the inner corona.

However, power spectra of magnetic field fluctuations in the solar wind, most likely of Alfvénic nature, are typically of the form $P_{B} \propto k^{-\eta}$, where $k$ is the wavenumber and $\eta$ the spectral index. Since observations yield $1.4 \leq \eta \leq 2.0$ (Bavassano et al. 1982), most of the power in the Alfvénic fluctuations is in the frequency range below the ion cyclotron frequency. Hence no substantial influence of these waves on ions can be expected (see Isenberg \& Hollweg 1983; Marsch et al. 1982). This difficulty can be overcome if the Sun launches waves in the ion cyclotron resonance range leading to the heating and acceleration of the solar wind (e.g., Axford \& McKenzie 1995; McKenzie et al. 1995, 1997; Tu \& Marsch 1997), or if there exists some mechanism which replenishes the resonant wave power as soon as it is absorbed by the particles.

To tap into the energy available in Alfvénic fluctuations, Hollweg (1986) assumed that a nonlinear cascade process, namely, the Kolmogorov dissipation of Alfvénic turbulence, transports energy from the low-frequency waves to the ion cyclotron resonant range, where it is picked up by the plasma through the cyclotron resonance. Hollweg \& Johnson (1988) extended this idea to a two-fluid solar wind model. Their results yielded the right qualitative features: a steep temperature rise in the transition region to over $10^{6} \mathrm{~K}$ in the corona and acceleration to supersonic speeds. These studies showed that the 
Kolmogorov dissipation rate is not capable of creating a transition region, a hot corona and a fast solar wind simultaneously, even though it seems that the mechanism is able to produce a fast solar wind (Isenberg 1990; Li et al. 1999).

Recent remote sensing observations from the Ultraviolet Coronagraph Spectrometer (UVCS) and Solar Ultraviolet Measurements of Emitted Radiation (SUMER) on board Solar and Heliospheric Observatory (SOHO) have enhanced our interests in the ion cyclotron resonance for coronal heating and solar wind acceleration. SUMER spectral line observations at the base of the corona in a southern coronal hole indicate that the temperature of minor ions decreases with increasing mass per charge (Tu et al. 1998). The temperature difference of various ions is more pronounced in the inner corona. UVCS observations reveal that ions not only have greater than massproportional temperatures in the inner corona, they are much hotter than electrons and are highly anisotropic as well (Kohl et al. 1998; Li et al. 1999; Cranmer et al. 1999). By recognizing the importance of the pumping by the second C II line in the interpretation of the UVCS observations, Li et al. (1998) concluded that the outflow speed of $\mathrm{O}^{+5}$ is about $400 \mathrm{~km} \mathrm{~s}^{-1}$ at $3 R_{S}$, already faster than protons, and these oxygen ions also have a large temperature anisotropy at the same distance. These recent advances strongly indicate that ion cyclotron resonance may indeed play an important role in the coronal heating (at least along open field lines), thus enhancing the interest in ion cyclotron resonance. Various models have been published to explain observations and make predictions using ion cyclotron resonance (see a thorough review by Hollweg \& Isenberg 2002 and the references).

One similar approach to tackle the lack of wave power at high frequencies in the solar wind is to include the non-linear interaction between outward propagating and inward propagating Alfvén waves. The non-linear interaction can result in a strong cascade. The idea was introduced by Tu et al. (1984). The cascade is able to explain the magnetic field spectrum evolution and to account for the proton heating beyond 10 solar radii (Tu 1987, 1988). In a series of papers, $\mathrm{Hu}$ and his coauthors applied this idea to the inner corona, and found that the cascade indeed is able to account for the heating and acceleration of fast solar wind (including minor ions) originating in coronal holes (Hu et al. 1999, 2000; Hu \& Habbal 1999). In these studies, a hot corona was treated as a boundary condition, the creation of a steep transition region and a hot corona were not considered. Although these turbulence cascade models are promising, it is still not clear what is the source of the necessary inward propagating waves and whether these inward propagating waves exist at all in the inner corona. It is also not clear if the non-linearity of waves in the corona is strong enough to produce cascades since wave amplitudes are small.

High frequency Alfvén waves have also been suggested (Axford \& McKenzie 1995), and been adopted to produce a steep transition region and a hot corona (Marsh \& Tu 1997a,b; Hackenberg et al. 2000), or to accelerate the fast solar wind (Tu \& Marsch 1997) by adopting a two fluid approach. Kinetic models have also been developed by assuming a relatively hot corona boundary (Vocks \& Marsch 2001, 2002). It has been found that plasma species may already lose thermal equilibrium at the very base of the corona or the top of transition region. The two fluid models developed by Hackenberg et al. (2000) can only reach 2 solar radii. The kinetic models developed by Vocks \& Marsch (2002) become numerically unstable at a few solar radii before reaching cruising speed and the preferential acceleration of minor ions is not accounted for. More recently, the above-mentioned two fluid transition region models were extended to a three fluid approach: the most abundant minor ions alpha particles were included in model calculations (Li 2002). It was shown that by heating alpha particles alone, a transition region, and a hot corona can be formed due to Coulomb coupling between alpha particles and protons, Coulomb coupling between protons and electrons, and the electron heat flux. Considering the strong evidence of ion cyclotron heating in the extended corona above polar coronal holes as mentioned above, one would naturally ask: can these high frequency Alfvén waves produce a self-consistent solution of a transition region, a hot corona and a fast wind if alpha particles are considered?

The goal of this paper is to develop a single coherent solution of a steep transition region, a hot corona and a fast solar wind stream using one single mechanism and including alpha particles. In this paper, it is assumed that an outward propagating power law spectrum of high frequency Alfvén waves (reaching ion cyclotron frequency) already exists below the transition region (Axford \& McKenzie 1995; Tu \& Marsch 1997). Physically, there are no "boundaries" between the corona and the solar wind. This one dimensional fluid model includes electrons, protons and alpha particles. A power law spectrum of Alfvén waves is assumed to supply all the energy needed to heat the corona and accelerate the solar wind. It is found that such a mechanism is capable of creating a steep transition region, a hot corona and a fast solar wind. Readers may also refer to the work by Hansteen \& Leer (1995), Hansteen et al. (1997) and Lie-Svendsen et al. (2002) who also considered chromosphere and ionization in their models. In those models, different heating mechanisms were used. In particular, Hansteen et al. (1997) have also included alpha particles in their models.

\section{Model description}

Standard three-fluid plasma transport equations for electrons, protons and alpha particles are considered, including heat conduction and radiation losses. We consider an isotropic, chargeneutral plasma. The one-dimensional model studied here is the same as those in Li et al. (1997) and Hu \& Habbal (1999), and the governing equations will not be repeated.

It is assumed that the magnetic flux tubes are strongly concentrated at the boundaries of the supergranule convection cells (Gabriel 1976; Marsch \& Tu 1997; Hackenberg et al. 2000; Li 2002). The plasma moves along the open magnetic field lines, and eventually builds up the solar wind. The flow tube cross section area is $a(r)=r^{2} f(r)$. We assume that the magnetic flux tubes in this paper have a two-step expansion:

$f(r)=f_{1}(r) f_{2}(r)$ 
and

$f_{i}(r)=\frac{f_{\mathrm{mi}} \mathrm{e}^{\left(r-r_{i}\right) / \sigma}+1-\left(f_{\mathrm{m} i}\right) \mathrm{e}^{\left(R_{\mathrm{S}}-r_{i}\right) / \sigma_{i}}}{\mathrm{e}^{\left(r-r_{i}\right) / \sigma_{i}}+1}, i=1,2$.

Here $f_{\mathrm{m} i}, r_{i}$, and $\sigma_{i}$ are constants. Parameters will be chosen such that $f_{1}(r)$ roughly represents a small scale expansion above the transition region, and the $f_{2}(r)$ represents the flux tube expansion beyond the solar disk. The first expansion reaches its full expansion at a height about $15000 \mathrm{~km}$, similar to the expansion factor used in the transition region and coronal heating models of Marsch \& Tu (1997a) and Li (2002). The expansion function is taken from Kopp \& Holzer (1976) for simplicity.

In this study the Coulomb $\log$ arithm $\ln \Lambda$ is taken to be 21 , it is assumed that plasmas in the transition region are collision dominated and classical thermal conduction parallel to the magnetic field can be used for the electrons, protons and alpha particles. However, the heat flux of protons rapidly becomes negligible with increasing heliocentric distances reflecting the collision-less nature of the proton gas (Li 1999; Olsen \& Leer 1999). To simulate this effect, we take

$$
\begin{aligned}
& \kappa_{\mathrm{e}}=7.8 \times 10^{-7} T_{\mathrm{e}}^{5 / 2}, \\
& \kappa_{\mathrm{p}}=3.2 \times 10^{-9} T_{\mathrm{p}}^{5 / 2} \exp \left[-\left(\frac{r-R_{\mathrm{S}}}{\xi R_{\mathrm{S}}}\right)^{2}\right], \\
& \kappa_{\alpha}=2 \times 10^{-11} T_{\alpha}^{5 / 2} \exp \left[-\left(\frac{r-R_{\mathrm{S}}}{\xi R_{\mathrm{S}}}\right)^{2}\right] .
\end{aligned}
$$

The parameter $\xi$ is taken to be 0.22 (however, the model results are found not sensitive to $\xi$ when it changes in the range $0.1 \sim 0.4)$. When the exponential factors in the above equations are dropped, they become the collision dominated heat flux conductivities (Spitzer 1962; Braginskii 1965).

The radiative energy loss in the electron energy equation is assumed to have the form parameterized by Rosner et al. (1978) for an optically thin medium. It strongly depends on the density of electrons.

When high frequency Alfvén waves propagate along open coronal magnetic field lines, ion cyclotron resonance may produce an upper limit $f_{\mathrm{H}}$ on the frequency of Alfvén waves by a sweeping mechanism (Marsch \& Tu 1997). Following Tu (1987) and $\mathrm{Hu} \&$ Habbal (1999), $f_{\mathrm{H}}$ is taken as

$$
f_{\mathrm{H}}=\alpha_{\mathrm{f}} \frac{f_{\alpha \mathrm{c}} v_{\mathrm{ph}}}{v_{\mathrm{A}}},
$$

where $f_{\alpha \mathrm{c}}$ is the gyro-frequency of alpha particles. The phase speed of an Alfvén wave is $v_{\mathrm{ph}}$. The $\alpha_{\mathrm{f}}$ is a constant less than unity, taken to be 0.7 in this study. This constant was taken to be $\leq 0.2$ in previous studies (Tu \& Marsch 1997; Hu \& Habbal 1999; Li 2002). At such a low frequency, alpha particles are not in resonance with ion cyclotron waves in a low beta plasma such as the one considered here. When $\alpha_{\mathrm{f}}=0.7$, ion cyclotron waves are already weakly dispersive. To avoid the complexity involved in the wave spectral evolution equation due to the wave dispersion (Eqs. (19), (20) and (21), Hu \& Habbal 1999), non-dispersive approximation is assumed.
The wave power spectrum density, $P(f, r)$, is related to the magnetic field variance $\left\langle\delta B^{2}\right\rangle$ and the Alfvén wave pressure $p_{\mathrm{w}}$ by

$$
\left\langle\delta B^{2}\right\rangle=8 \pi p_{\mathrm{w}}=\int_{f_{\mathrm{L}}}^{f_{\mathrm{H}}} P \mathrm{~d} f .
$$

It is assumed that the Alfvén waves originating below the transition region have a minimum frequency $f_{\mathrm{L}}$ and a maximum frequency $f_{\mathrm{d}}$. If $f_{\mathrm{d}}<f_{\mathrm{H}}$, these Alfvén waves do not resonate with ions, and no wave heating will result. In such a case, $p_{\mathrm{w}}$ will be related to the wave power spectrum $P(f, r)$ as

$$
p_{\mathrm{w}}=\frac{\int_{f_{\mathrm{L}}}^{f_{\mathrm{d}}} P \mathrm{~d} f}{8 \pi} \text {. }
$$

Following Marsch \& Tu (1997a), it is also assumed that $P(f) \propto f^{-1}$, which was suggested by observations in the ecliptic near 0.3 AU (Bavassano et al. 1982; Marsch \& Tu 1990) and over the poles (Horbury et al. 1995).

The treatment of the wave spectral evolution closely follows Hu \& Habbal (1999), however non-linear cascade is ignored in this study. The plasma in a magnetic flux tube is only heated by a sweeping mechanism (Marsch \& Tu 1997a). The total dissipation rate from this mechanism is divided between the acceleration and heating for different ion species determined by the microphysics of the resonant interaction. For a Maxwellian distribution of a thermally isotropic plasma interacting with a spectrum of parallel left-hand-polarized ion cyclotron waves, the resonant acceleration and heating rate of species $i$ are given by Dusenberg \& Hollweg (1981), Marsch et al. (1982) and also Hu \& Habbal (1999). In the evaluation of the integrals involved in the quasi-linear terms, the wave power spectrum and the dispersion relation in the dissipation range must be known. Due to the strong magnetic field in the transition region, the plasma beta value is small. Hence cold plasma approximation for the dispersion of these waves can be adopted. Since ion cyclotron resonance is very efficient in dissipating wave power in small scales, $P(k) \propto k^{-5}$ is assumed in this study. It will be shown, in the transition region and near Sun region, that the outflow velocity difference between alpha particles and protons is small compared with Alfvén speed, as cold plasma dispersion effectively prohibits cyclotron resonance between waves and protons.

The time-dependent equations of the three-fluid plasma system are numerically integrated with variable step sizes in space and time using a fully implicit scheme described by $\mathrm{Hu}$ et al. (1997) and Li et al. (1997). Model calculations start from a point in the lower transition region where the temperature is $6 \times 10^{4} \mathrm{~K}$ and extend to $50 R_{\mathrm{S}}$. The radial step $\mathrm{d} r$ gradually increases from $35 \mathrm{~m}$ at the lower boundary, to about $0.8 R_{\mathrm{S}}$ at the top of the computational domain, and 1100 grid points are used in calculations. The maximum error in any of the conserved quantities is less than $1 \%$ once a steady state is reached.

\section{Results}

In this section, model calculations are presented. Calculations start at $T_{\mathrm{e}}=T_{\mathrm{p}}=T_{\alpha}=6 \times 10^{4} \mathrm{~K}$. The starting temperature is chosen so that both hydrogen and helium are almost 
Table 1. Conditions at the base of an open magnetic flux and flux geometry.

\begin{tabular}{ll}
\hline \hline Temperature $\left(T_{\mathrm{e}}=T_{\mathrm{p}}=T_{\alpha}\right)$ & $6 \times 10^{4} \mathrm{~K}$ \\
Alpha particle abundance $\left(n_{\alpha} / n_{\mathrm{p}}\right)$ & 0.06 \\
Electron density $\left(10^{9} \mathrm{~cm}^{-3}\right)$ & 3.8 \\
Wave amplitude $(\delta V)$ & $21 \mathrm{~km} \mathrm{~s}^{-1}$ \\
$r_{1}$ & $1.004 R_{\mathrm{S}}$ \\
$\sigma_{1}$ & $0.005 R_{\mathrm{S}}$ \\
Area expansion factor $\left(f_{\mathrm{m} 2}\right)$ & 7 \\
$r_{2}$ & $1.31 R_{\mathrm{S}}$ \\
$\sigma_{2}$ & $0.51 R_{\mathrm{S}}$ \\
Low frequency bound $\left(f_{\mathrm{L}}\right)$ & $10^{-2} \mathrm{~Hz}$ \\
\hline
\end{tabular}

fully ionized, and complications involved with ionization can be avoided (Hansteen et al. 1997). The corresponding electron density at the boundary is $3.8 \times 10^{9} \mathrm{~cm}^{-3}$. The adopted electron thermal pressure is $n_{\mathrm{e}} T_{\mathrm{e}}=2.3 \times 10^{14} \mathrm{~K} \mathrm{~cm}^{-3}$. This pressure is typical in coronal holes according to (Warren \& Hassler 1998). Warren \& Hassler (1998) found that thermal pressure is roughly a constant $\left(\log n_{\mathrm{e}} T_{\mathrm{e}}=14.2\right)$ in the transition region when the electron temperature varies from $2 \times 10^{4}$ to $10^{6} \mathrm{~K}$. Our computations end at 52.5 solar radii. At 52.5 solar radii, the fast solar wind should be almost fully developed, and beyond 10 solar radii, the cascading effect may not be ignored (Tu 1987). Three models will be presented in this study, some common parameters of these models at the base of the open magnetic flux tube and the flux tube geometry are listed in Table 1.

\subsection{Strong magnetic flux concentration}

We first present model calculations with strong magnetic flux concentration in the supergranule convection zone boundaries. Parameters at the lower computation boundary used in this study are listed in Table 1 . The flux tube geometry parameters are as follows: $f_{\mathrm{m} 1}=13$ is the maximum expansion factor of the cross-section of a flux tube in the transition region, $r_{1}=1.004 R_{\mathrm{S}}$ is the heliocentric distance at which the major expansion takes place. The parameter $\sigma_{1}=0.005 R_{\mathrm{S}}$ represents the length over which the expansion occurs. This first super-radial expansion is complete by about $15000 \mathrm{~km}$. The parameters for the second super-radial expansion are: $f_{\mathrm{m} 2}=7$, $r_{2}=1.31 R_{\mathrm{S}}$ and $\sigma_{2}=0.51 R_{\mathrm{S}}$. This expansion is complete at about $3 R_{\mathrm{S}}$, above $3 R_{\mathrm{S}}$, the solar wind is almost radial.

The magnetic field at the bottom of the funnel is $123.5 \mathrm{G}$. This means that we assume the coronal funnels considered here may originate from deep in the photosphere (Dowdy et al. 1986). The low-frequency end of the wave spectrum is fixed at $f_{\mathrm{L}}=10^{-2} \mathrm{~Hz}$. The wavelength of the waves below this frequency is very large compared to the scale height in the transition region and corona. Hence those waves are ignored. The high-frequency end of the wave spectrum $f_{\mathrm{d}}$ will be treated as a free parameter. This parameter determines where ion cyclotron resonance occurs in a flux tube, as can be seen from Fig. 1.

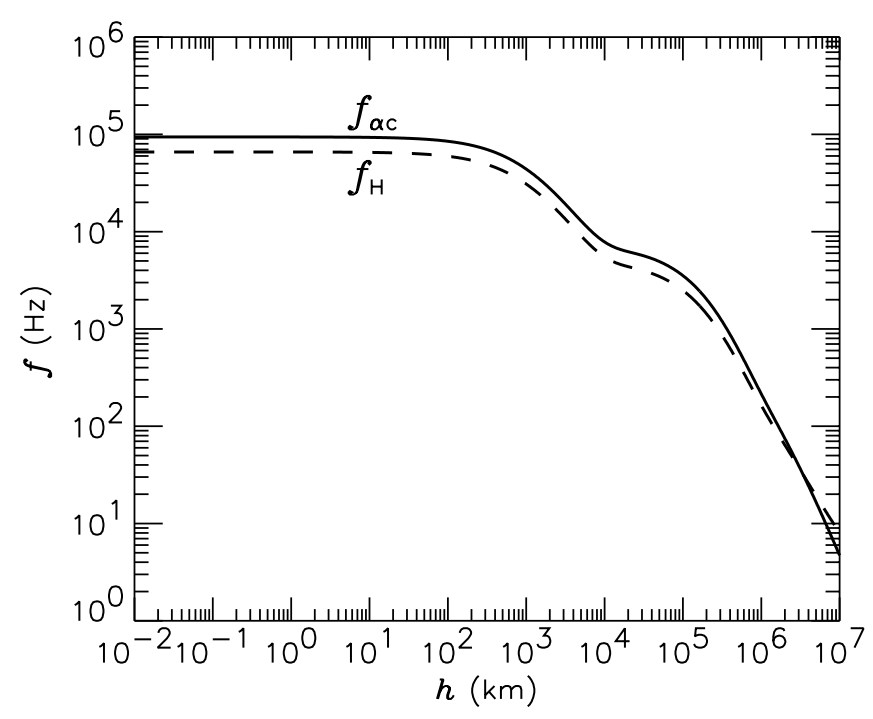

Fig. 1. Gyro-frequency of alpha particles in a flux tube, the curve for $f_{\mathrm{H}}$ (dashed line) is taken from model 1 in Fig. 2.

Figure 1 shows the gyro-frequency of alpha particles $f_{\alpha \mathrm{c}}$ and $f_{\mathrm{H}}$ as a function of height $h$, which is defined by $h=r-r_{0}$, and where $r_{0}$ is the heliospheric distance at the lower boundary (assumed to be $R_{\mathrm{S}}$ ). Below $10^{5} \mathrm{~km}$, flow velocities are much smaller than the Alfvén speed and $v_{\mathrm{ph}}$ is nearly identical to $v_{\mathrm{A}}$. As a result, $f_{\mathrm{H}} \sim 0.7 f_{\alpha \mathrm{c}}, 0.7 f_{\alpha \mathrm{c}}$ is roughly the frequency at which cyclotron resonance transfers the wave energy to alpha particles. However, $f_{\mathrm{H}}$ can be Doppler shifted and become larger than $f_{\alpha \mathrm{c}}$ at larger distances. If $f_{\mathrm{d}}<f_{\mathrm{H}}$, no wave heating will occur but the waves can still transfer momentum to the plasma (via wave pressure gradient). The abundance of alpha particles is chosen at $6 \%$. This abundance is slightly higher than its in situ measured value of about $5 \%$ in fast solar wind streams, but lower than the helium abundance at the photosphere. Previous solar wind models showed that this abundance at the coronal base can often lead to observed helium abundance in interplanetary space ( $\mathrm{Li}$ et al. 1997; Hu \& Habbal 1999).

A steady state solution for the model calculations is shown in Fig. 2 (model 1). $f_{\mathrm{d}}$ is taken to be $50000 \mathrm{~Hz}$, so the wave heating starts at $h=300 \mathrm{~km}$ (see Figs. 1 and 3). Ion cyclotron waves indeed produce a very rapid temperature increase, a hot corona and a fast solar wind: the speed of protons and alphas is $767 \mathrm{~km} \mathrm{~s}^{-1}$ and $826 \mathrm{~km} \mathrm{~s}^{-1}$ at 52.5 solar radii, respectively. The particle flux of protons is $2.4 \times 10^{8} \mathrm{~cm} \mathrm{~s}^{-1}$, scaled to 215 solar radii ( $1 \mathrm{AU})$, a typical observed value in the fast solar wind at 1 AU (Phillips et al. 1995). The initial heating of alpha particles is extremely strong, the temperature of alpha particles already reaches $2.35 \times 10^{6} \mathrm{~K}$ at the very bottom (which is shown more clearly in Fig. 3). The heating rate of alpha particles $Q_{\alpha}$ declines by three orders of magnitude in a narrow region. Roughly speaking, only alpha particles are heated below $2 R_{\mathrm{S}}$, proton heating $\left(Q_{\mathrm{p}}\right)$ is negligible in the same region. However, the proton temperature $T_{\mathrm{p}}$ still increases substantially due to the Coulomb coupling. After $1.4 R_{\mathrm{S}}$, the Coulomb coupling between protons and alpha particles is not strong enough to balance the adiabatic cooling of protons, and $T_{\mathrm{p}}$ decreases. 

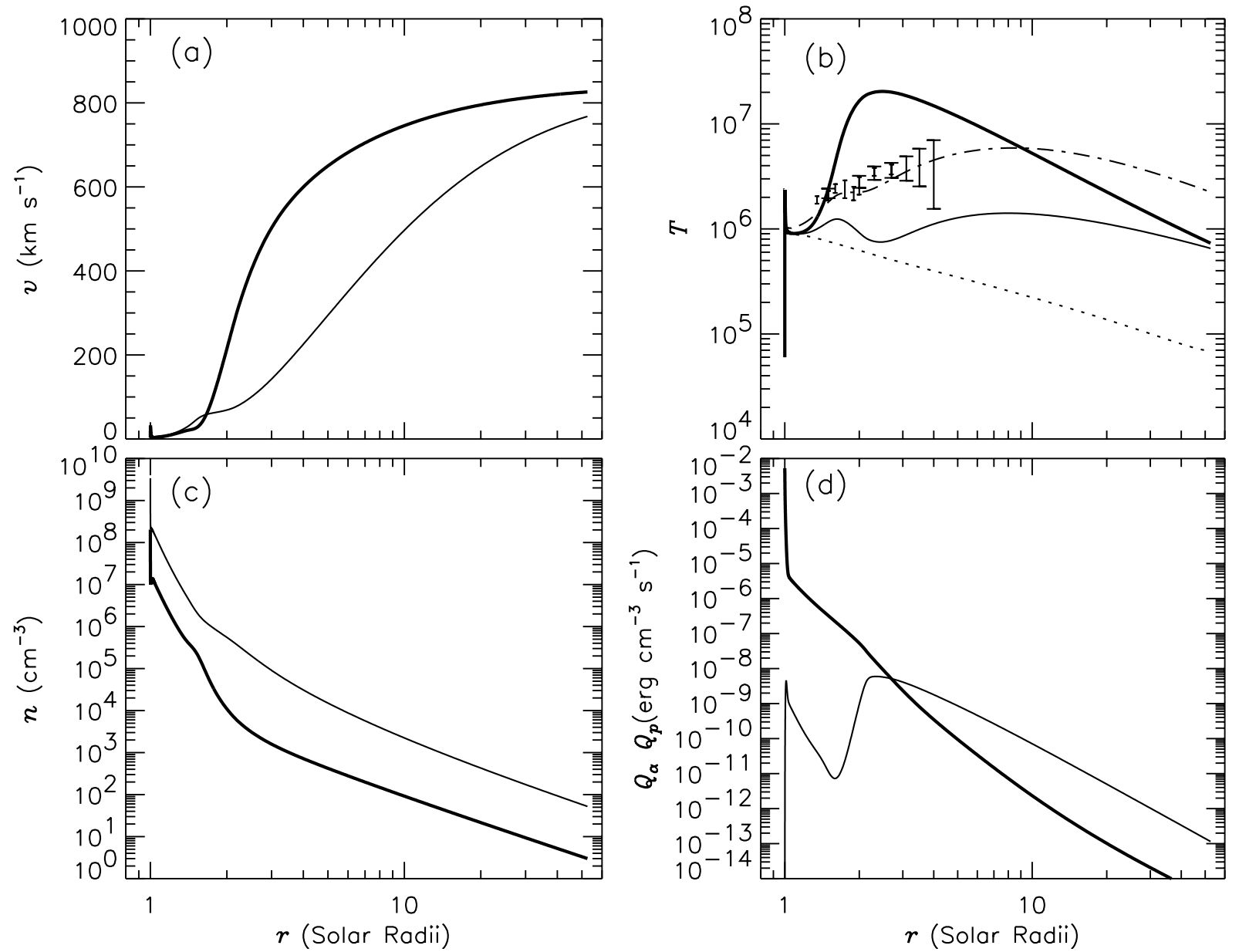

Fig. 2. A coronal heating and fast solar wind model in which the plasma is heated by ion cyclotron waves, Model 1. a) Speed of protons (thin solid line) and alpha particles (thick solid line). b) Temperature of electrons (dotted line), protons (thin solid line) and alpha particles (thick solid line). The dashed-dotted line is the proton effective temperature calculated from model 1. The narrow and wide vertical bars represent the observed proton effective temperatures from UVCS observations reported by Esser et al. (1999) and Kohl et al. (1998), respectively. c) Density of protons (thin solid line) and alpha particles (thick solid line). d) Heating rates of protons (thin solid line) and alpha particles (thick solid line). Here $f_{\mathrm{d}}=50000 \mathrm{~Hz}$.

Beyond $2 R_{\mathrm{S}}$, alpha particles are fast, and start partially to lose resonance with waves, and protons soon absorb more energy from waves than do the alpha particles. At $2.5 R_{\mathrm{S}}$, the wave heating of protons finally counterbalances adiabatic cooling and $T_{\mathrm{p}}$ increases. Alpha particles gradually lose resonance with waves and $Q_{\alpha}$ becomes very small above $3 R_{\mathrm{S}}$.

The model calculation predicts a rather low proton temperature between 2 and 4 solar radii. The local minimum $T_{\mathrm{p}}$ at $2.5 R_{\mathrm{S}}$ is only $7.5 \times 10^{5} \mathrm{~K}$, only slightly higher than the electron temperature. However, the Alfvén wave amplitude is strong (as will be shown in Fig. 6) in the region. Hence, the effective proton temperature

$T_{\text {eff }}=T_{\mathrm{p}}+\frac{m_{\mathrm{p}}\left\langle\delta V^{2}\right\rangle}{2 k_{\mathrm{B}}}$

is still significant in the same distances (see the dashed-dotted line in Fig. 2b). Here $k_{\mathrm{B}}$ is the Boltzmann constant, $m_{\mathrm{p}}$ is the proton mass. The narrow vertical bars represent the observed proton effective temperatures from UVCS spectral line width measurements in a northern polar coronal hole reported by
Esser et al. (1999), while the wide vertical bars represent similar observations in polar coronal holes reported by Kohl et al. (1998). All these observations were made when the Sun was at its phase of minimum activity. In general, the calculated proton effective temperature matches observations reasonably well. Beyond $2 R_{\mathrm{S}}$, the proton temperature is low, but the calculated proton effective temperature is still very close to the observed lowest effective temperatures or within the observed uncertainties. Remember, the calculated effective proton temperature from Model 1 does not include the line-width broadening due to the outflow velocities along the line of sight, and the ion temperature anisotropies are neglected. Also note, in a twofluid solar wind model, the proton effective temperatures from model calculations are at the upper limit of observed proton effective temperatures ( $\mathrm{Li}$ et al. 1999). Future work to include species temperature anisotropies may improve the match with observations. This study suggests that the hydrogen Lyman alpha lines observed by UVCS may be primarily broadened by Alfvén waves, while spectral lines of minor ions may be primarily broadened by thermal motions and outflow velocities 

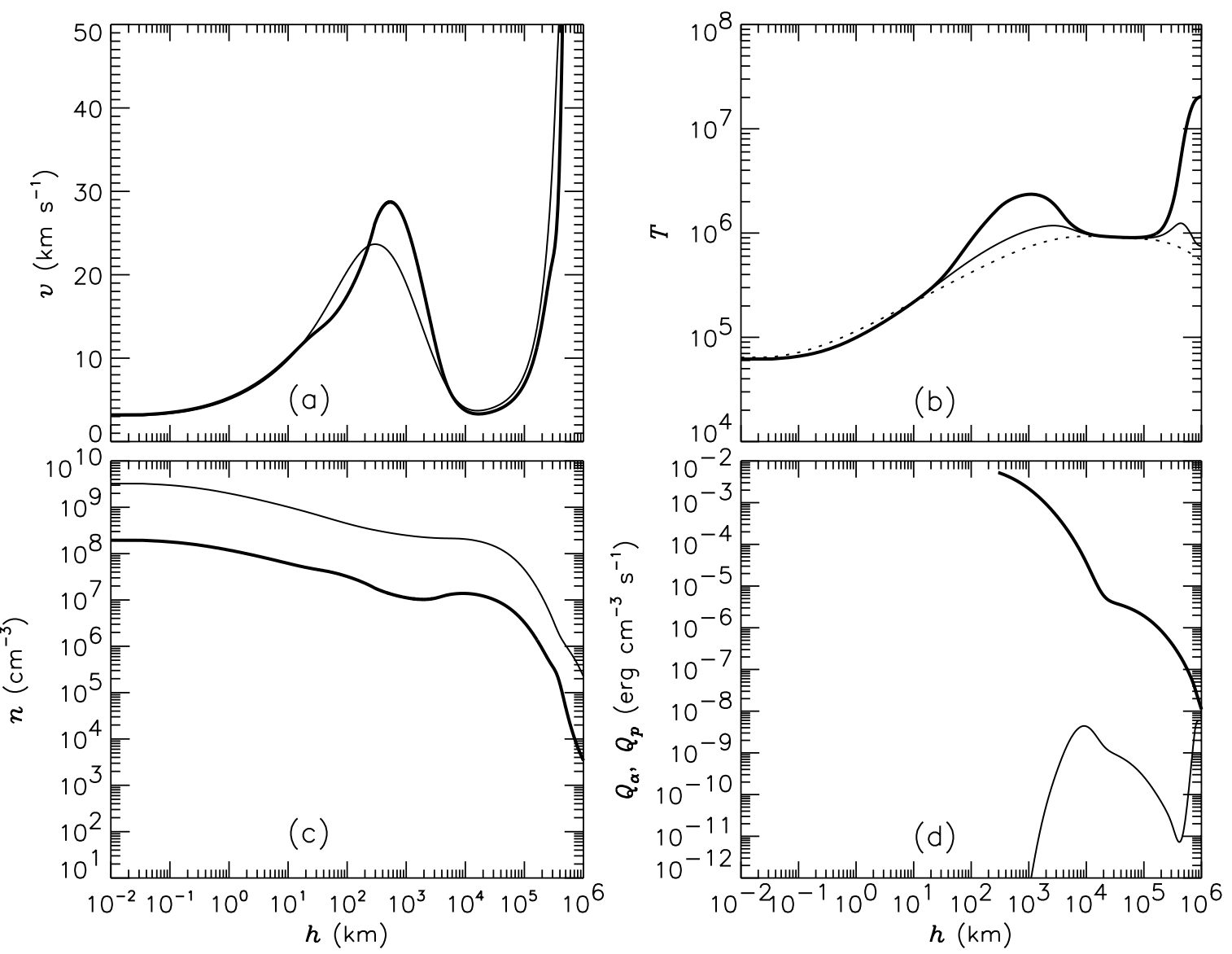

Fig. 3. An enlarged version of Fig. 2 in the region just above the lower boundary.

along the line of sight. The transverse velocity disturbance $\delta V_{i}$ of ion species $i$ with outflow velocity $v_{i}$ is (Isenberg \& Hollweg 1982)

$\delta V_{i}=\frac{v_{\mathrm{ph}}-v_{i}}{v_{\mathrm{ph}}} \delta V$

At 2-4 solar radii, minor ion velocity $v_{\mathrm{ph}}-v_{i}$ may be a significant fraction of Alfvén phase velocity $v_{\mathrm{ph}}$ (for protons, the ratio $\left(v_{\mathrm{ph}}-v_{\mathrm{p}}\right) / v_{\mathrm{ph}}$ is roughly 1$)$. Hence the line-width broadening of minor ions from waves is weakened. The alpha particles lose resonance with Alfvén waves at large distances, suggesting alpha particles (and perhaps other minors too) must be heated by other mechanisms in interplanetary space (plasma microinstabilities may be the main sources).

Note the wave amplitude at the base of the flux tube is $21 \mathrm{~km} \mathrm{~s}^{-1}$, much smaller than the value used in $\mathrm{Li}$ (2002). The wave amplitude is at the lower end observed by SUMER (Tu et al. 1998). However SUMER observations were done above the solar limb. The coronal funnels considered in this paper are deeply rooted in the lower transition region, a smaller wave amplitude can be justified due to the high density. By combining the solar wind acceleration and the transition region in a full treatment, the heat deposited below the subsonic point can increase mass fluxes substantially. To produce a particle flux constrained by in situ measurements, a smaller wave amplitude is necessary. This demonstrates that the coronal heating along open field lines and the solar wind acceleration must be treated as a single problem.

To show the transition region and the initial heating process more clearly, Fig. 3 shows a small region above the lower boundary of the solution in Fig. 2. When temperatures are still low, alpha particles and protons have virtually the same speed $(h<30 \mathrm{~km})$. At $200 \mathrm{~km}<h<6000 \mathrm{~km}$, alpha particles are not only faster, but much hotter than protons as well. The proton velocity at the lower boundary is $v_{\mathrm{p}}=3 \mathrm{~km} \mathrm{~s}^{-1}$. The alpha particles reach a local maximum velocity of $28 \mathrm{~km} \mathrm{~s}^{-1}$ at a height of $600 \mathrm{~km}$. This effect is totally due to the introduction of ion cyclotron resonance. Below this point, the wave frequency at the high end of the spectrum is still smaller than the frequency where alpha particles can resonate with the waves. Hence no wave dissipation occurs. The most interesting feature of Fig. 3 is that at the top of the transition region or at the bottom of the corona, alpha particles and protons (and electrons) can be significantly away from thermal equilibrium. In the region $30 \mathrm{~km}<h<300 \mathrm{~km}$, alpha particles are slower than protons. This is because the higher temperature gradient of alpha particles (compared with protons) actually produces a negative pressure gradient force for alpha particles (see Fig. 5), and alpha particles are slower than protons. Since $Q_{\mathrm{p}}$ is negligible at $h<10^{5} \mathrm{~km}$, protons and electrons are heated by Coulomb coupling with protons and alpha particles, and by the electron heat flux. The evolution of speed and temperatures in Fig. 3 is similar to the results in Hackenberg et al. (2000). The loss of 


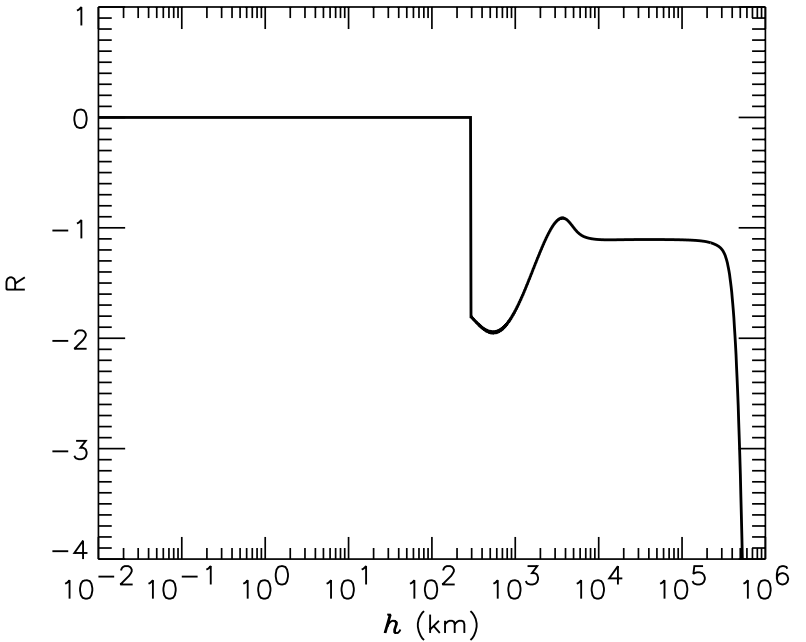

Fig. 4. Ratio between alpha particle heating term due to waves and collisional heating terms in alpha particle energy energy equation $(\mathrm{Hu}$ \& Habbal 1999).

thermal equilibrium between protons and electrons at the top of transition region or the bottom of the corona has also been found by Marsch \& Tu (1997a) and Vocks \& Marsch (2002). Here it is shown that protons and alpha particles can also lose thermal equilibrium in the same region. The transition region of model 1 is very narrow: the jump of the electron temperature $T_{\mathrm{e}}$ from $6 \times 10^{4}$ to $6 \times 10^{5} \mathrm{~K}$ takes place in $390 \mathrm{~km}$.

A few comments can be made about the velocity profile of alpha particles in Fig. 3a. Transition region spectral line observations often find various velocities of ions. These velocities can vary from a few to about twenty kilometers per second. For instance, Hassler et al. (1999) found that in network boundaries an average outflow velocity is several kilometers per second when $T>6.3 \times 10^{5} \mathrm{~K}$, while Wilhelm et al. (2000) estimated that the outflow velocity of $\mathrm{Ne}^{7+}$ is about $14 \mathrm{~km} \mathrm{~s}^{-1}$ along open magnetic field lines from their SUMER data. The velocity of alpha particles $v_{\alpha}$ in Fig. $3 \mathrm{a}$ is in the range $3 \mathrm{~km} \mathrm{~s}^{-1}<v_{\alpha}<28 \mathrm{~km} \mathrm{~s}^{-1}$ at different heights. Even though no other minor ions have been included in this investigation, however, if those ions behave similar to the alpha particles, their velocity is expected to vary in a similar manner. Hence, if spectral lines are observed at different heights, observed outflow velocities may vary accordingly.

The fact that minor ions can flow much faster than the bulk plasma flow in the upper transition region may have implications on the analysis of transition region spectral line observations. Traditional atomic physics calculations on which the diagnostics of both temperatures and densities depend often assume equal ion and electron temperatures. From Doppler shift measurements of transition region spectral lines, Dere et al. (1989) found that the mass flux in the region is more than enough to account for in situ measured solar wind mass flux. If minor ions are indeed much faster than protons and electrons in the transition region, one cannot safely determine the bulk outflow velocity of plasmas in the region by using minor ion outflow velocities.

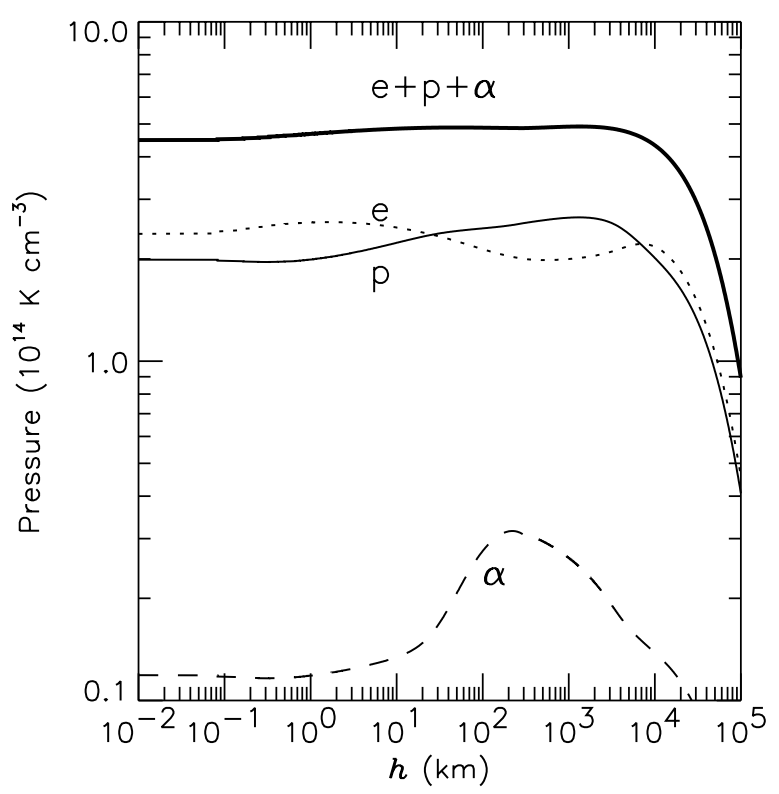

Fig. 5. Model 1: thermal pressure of electrons (dotted line), protons (thin solid line), and alpha particles (dashed line). The thick solid line represents the total pressure $n_{\mathrm{e}} T_{\mathrm{e}}+n_{\mathrm{p}} T_{\mathrm{p}}+n_{\alpha} T_{\alpha}$.

Needless to say, in the region where no direct external heating is added, the strong heating of the three species is entirely due to the heat fluxes of the species and Coulomb coupling between them. The Coulomb collisional coupling between ions is much stronger than the coupling between ions and electrons. Hence protons and alpha particles have identical temperatures. When the electron temperature gradient becomes very large, electrons become hotter than protons and alpha particles ( $h<10 \mathrm{~km}$, Fig. 3b). This can be easily understood since the classical electron thermal conductivity is more than one magnitude larger than the classical ion thermal conductivities. Note that the strong Coulomb coupling makes the temperature difference less than $20 \%$ (at $h<10 \mathrm{~km}$ ). This seems to make our assumption that species have the same temperature at $T_{\mathrm{e}}=6 \times 10^{4} \mathrm{~K}$ questionable. However, the electron heat conductivity is proportional to $T_{\mathrm{e}}^{5 / 2}$, the heat flux is relatively small at low temperatures. And most importantly, Coulomb collisions become extremely strong at low temperatures. This small discrepancy does not influence the model solution in a significant way. To avoid this discrepancy, future models need to include the chromosphere and the detailed ionization processes of hydrogen and helium, which is beyond the scope of this paper.

To show how the loss of thermal equilibrium between protons and alpha particles can occur around $h=1000 \mathrm{~km}$ in the model, $R$, the ratio between $Q_{\alpha}$ and proton/alpha Coulomb coupling terms in the energy equation of the alpha particles, is plotted in Fig. 4. Clearly only in the region where $|R|$ is significantly larger than 1 do alpha particles lose thermal equilibrium with protons. Note the rate $R$ is negative, suggesting that alpha particles lose thermal energy to protons due to collisions. At $h<10^{5} \mathrm{~km}$, roughly speaking, only alpha particles are directly heated by waves. Without any other external heat sources, protons are only heated via Coulomb coupling with alpha particles. Above $h=300000 \mathrm{~km}$ (or about $1.4 R_{\mathrm{S}}$ ), this ratio is 

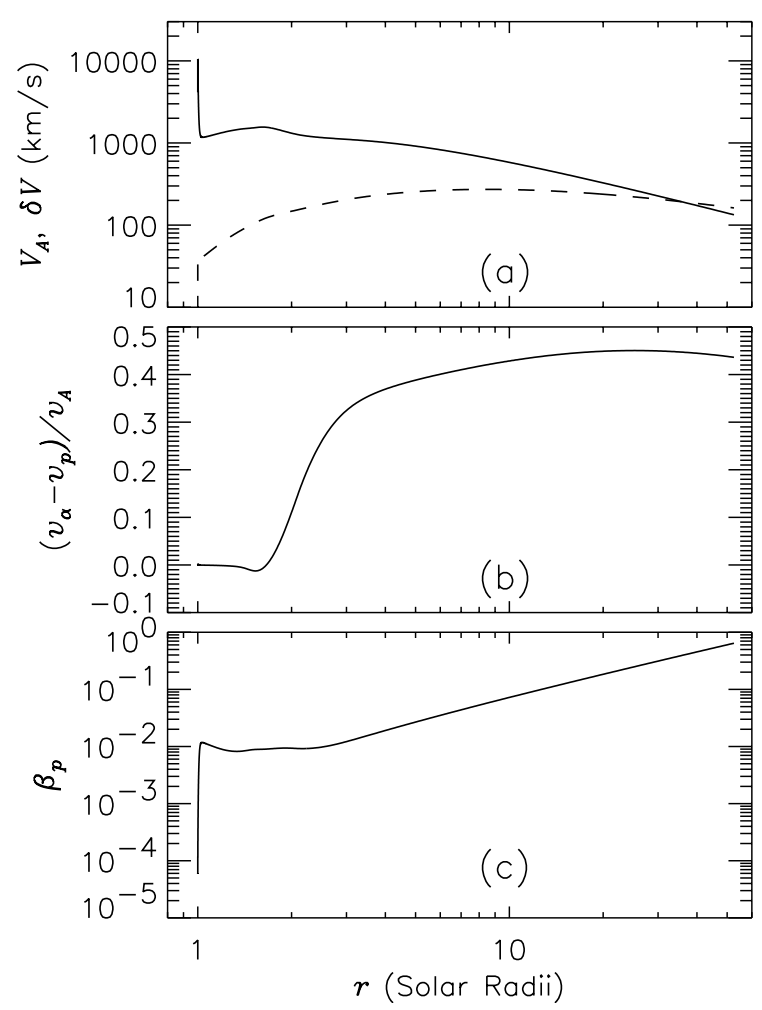

Fig. 6. Model 1: a) the Alfvén speed (solid line) and the wave amplitude (dashed line); b) ratio $\left.\left(v_{\alpha}-v_{\mathrm{p}}\right) / v_{\mathrm{A}} ; \mathbf{c}\right)$ Proton beta value.

much larger than 1 , suggesting that Coulomb coupling is not important.

Figure 5 shows the species thermal pressures from the model in Fig. 2. The total thermal pressure is nearly a constant between $T_{\mathrm{e}}=6 \times 10^{4} \mathrm{~K}$ and $T_{\mathrm{e}} \simeq 9 \times 10^{6} \mathrm{~K}$. However, the individual species pressures are not as constant. Ion cyclotron resonance heating yields a large alpha thermal pressure bump at $20 \mathrm{~km}<h<10000 \mathrm{~km}$.

The Alfvén speed $v_{\mathrm{A}}$ and Alfvén wave amplitude $\delta V=$ $\sqrt{2 p_{\mathrm{w}} / \rho}$ are shown in Fig. 6a. Here $\rho$ is the total plasma mass density. The Alfvén wave amplitude reaches $35 \mathrm{~km} \mathrm{~s}^{-1}$ at $1.014 R_{\mathrm{S}}$, and $n_{\mathrm{e}}=2.3 \times 10^{8} \mathrm{~cm}^{-1}, T_{\mathrm{e}}=9.3 \times 10^{5} \mathrm{~K}$. The ratio $\left(v_{\alpha}-v_{\mathrm{p}}\right) / v_{\mathrm{A}}$ is shown in Fig. 6b. To compare Fig. $6 \mathrm{~b}$ with Fig. 2d, the proton heating rate $Q_{\mathrm{p}}$ becomes larger than $Q_{\alpha}$ at about $2.7 R_{\mathrm{S}}$, where the ratio $\left(v_{\alpha}-v_{\mathrm{p}}\right) / v_{\mathrm{A}} \approx 0.29$. Note, if warm plasma dispersion relation is used, protons may get more heating faster (Li \& Habbal 1999). Hollweg \& Isenberg (2002) also discussed this issue. The "switch" from primarily heating alpha particle to primarily heating protons depends on the abundance of alpha particles, the plasma beta value and the detailed dissipation range spectrum. The proton beta value, the ratio between the proton thermal pressure and magnetic pressure

$\beta_{\mathrm{p}}=\frac{2 k_{\mathrm{B}} T_{\mathrm{p}}}{m_{\mathrm{p}} v_{\mathrm{A}}^{2}}$

is plotted in Fig. 6c. This value is small at $r<10 R_{\mathrm{S}}$.

Figure 7 shows the various contributions to the energy flux, scaled to the inner boundary. The total energy flux is a sum of the Alfvén wave energy flux; kinetic energy flux of protons and alpha particles; gravitational energy flux; total enthalpy flux;

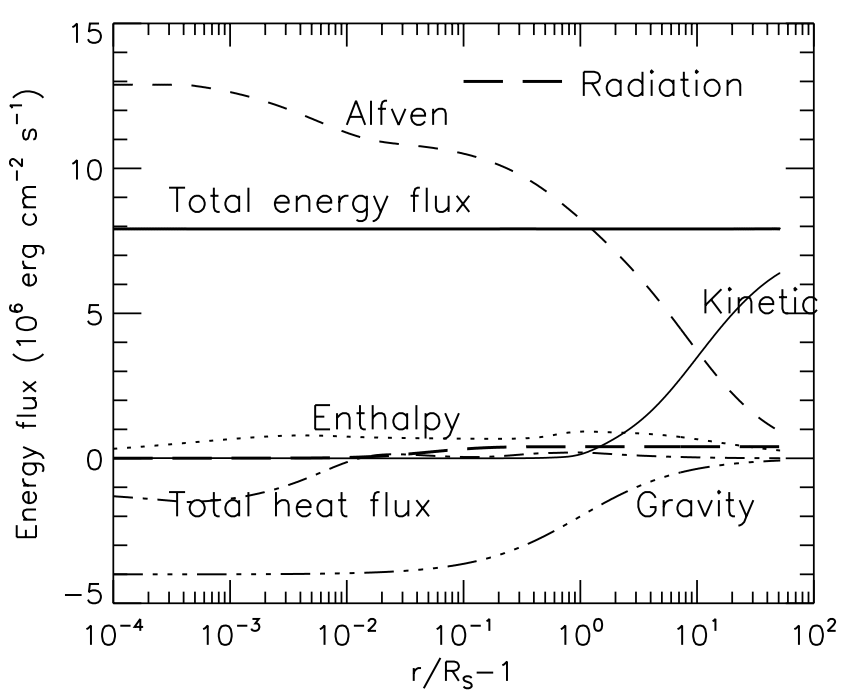

Fig. 7. Contributions to the energy flux density, multiplied by $a(r) / a\left(R_{\mathrm{S}}\right)$ for Model 1: Alfvén wave energy flux (dashed); total enthalpy (dotted); total kinetic energy flux (thin solid); total heat flux (dot-dashed); gravity (dash-triple-dotted); radiation (thick dashed). The thick solid line is the total energy flux density.

total heat flux of electrons, protons and alpha particles; and the radiation loss (an integration from $1 R_{\mathrm{S}}$ to a given distance). It is obvious that the radiation loss is a small quantity in the energy budget. The heat flux is only important in the transition region. The total energy flux is a straight horizontal line, indicating an excellent energy conservation. In fact, the maximum relative error in the total energy flux is $0.16 \%$, which is too small to be seen in Fig. 7.

From Figs. 3 and 4, it is seen that alpha particles lose thermal equilibrium with protons around $h=1000 \mathrm{~km}$ when the heat gain that the alpha particles obtained from waves is much more than the heat loss through the Coulomb coupling with protons. In this region, $f_{\mathrm{H}}$ decreases most rapidly with height due to the fast flux tube expansion. If the wave heating begins at an even higher location in the coronal funnel, for instance, the wave heating starts after the first flux tube expansion almost ends, the heating rate $Q_{\alpha}$ will be smaller since $\mathrm{d} f_{\mathrm{H}} / \mathrm{d} r$ now is smaller and there is less wave energy at the high frequency end. One of course expects that the loss of an equilibrium between alpha particles and protons is going to be weaker. This is exactly the case when the wave frequency at the high end of the spectrum $f_{\mathrm{d}}$ is $17000 \mathrm{~Hz}$. The overall feature of this case is similar to those in Fig. 2 and will not be shown here. The region just above the lower boundary is shown in Fig. 8 . The ion cyclotron heating is not introduced until at a height $2070 \mathrm{~km}$ above the lower boundary (Fig. 8d). Even though the wave heating still leads to different alpha particle and proton temperatures at $2000 \mathrm{~km}<h<4000 \mathrm{~km}$ (Fig. 8d), the difference is far less significant than in Fig. 3b. There is virtually no velocity difference in this region. Plasma species are roughly in thermal equilibrium at $h<10^{5} \mathrm{~km}$ (Figs. $8 \mathrm{a}$ and 8b). The temperatures of Model 2 in the transition region below $5000 \mathrm{~km}$ are much lower than in model 1 (see Figs. $3 \mathrm{~b}$ and $7 \mathrm{~b}$ ). However, Model 2 has a slightly lower maximum electron temperature $\left(8.34 \times 10^{5} \mathrm{~K}\right.$, compared to $9.31 \times 10^{5} \mathrm{~K}$ in model 1$)$. 

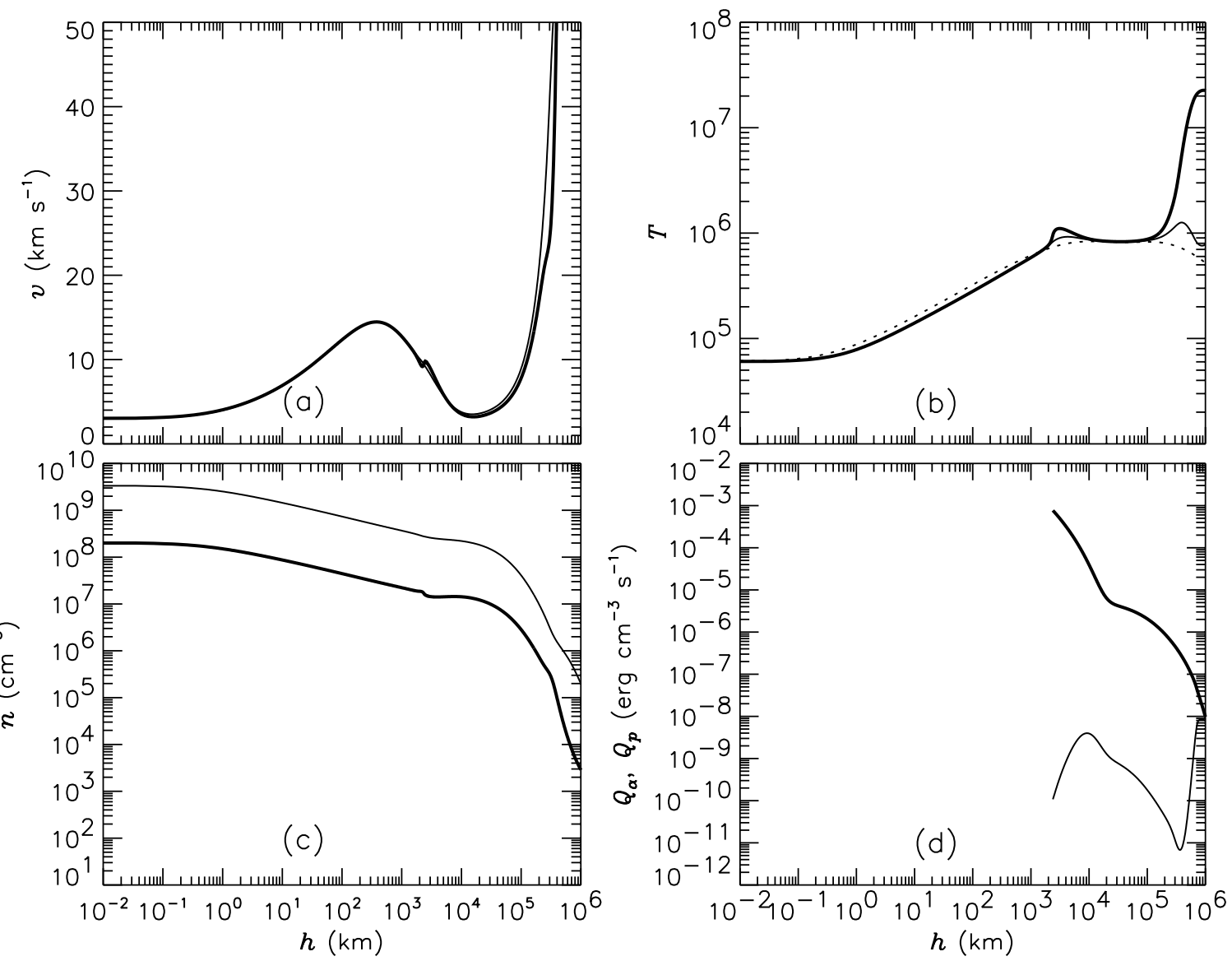

Fig. 8. A small region just above the lower boundary for Model 2. Here $f_{\mathrm{d}}=17000 \mathrm{~Hz}$.

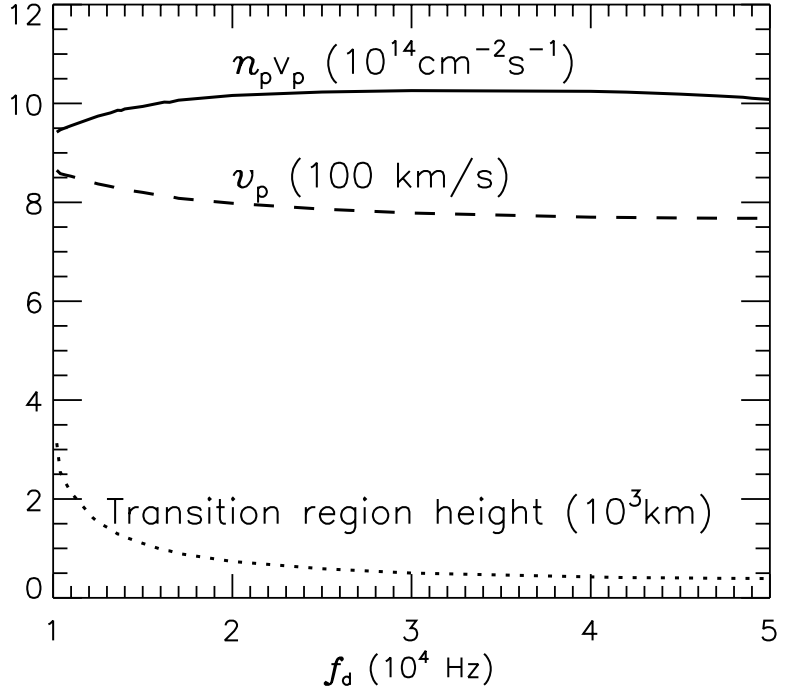

Fig. 9. Proton particle flux density (solid line) at the lower computation boundary, proton speed (dashed line) at $52.5 R_{\mathrm{S}}$, and the transition region height (see text), as a function of $f_{\mathrm{d}}$.

Although electrons are not directly heated, these maximum $T_{\mathrm{e}}$ match SUMER observations in coronal holes (Wilhelm et al. 1998). Overall, the difference between Model 1 and Model 2 is marginal except in the transition region.
Figure 9 shows the proton particle flux density at $1 R_{\mathrm{S}}$, the proton velocity at $52.5 R_{\mathrm{S}}$, and the height of the transition region (defined as the distance over which the electron temperature increases from $6 \times 10^{4}$ to $6 \times 10^{5} \mathrm{~K}$ ), as a function of $f_{\mathrm{d}}$, other parameters are the same as used in Model 1. Once the wave amplitude at the lower boundary and the magnetic field geometry are chosen, the total energy flux entering the computational domain is fixed (the only energy losses are heat flux and radiation loss). However, if $f_{\mathrm{d}}$ is smaller than $10000 \mathrm{~Hz}$, a steady state solution can not be obtained. The reason is that as $f_{\mathrm{d}}$ is small, the electron temperature gradient becomes smaller. The transition region becomes too wide so the electron heat flux is unable to balance the local radiation loss. Hence a steady state hot corona and wind cannot be found. (Note, however, if the chromosphere is also included in the model calculation, the position of transition region may change as the position of the energy deposit changes. But this is beyond the scope of the present study.) Of course, if the wave power at high frequency is enhanced, as suggested by Tu \& Marsch (1997), the result may be different. Roughly speaking, the proton mass flux is almost constant (the change is less than 10\%), although the plasma parameters at the coronal base can be very different as shown in Figs. 3 and 8. The small variation reflects the different heat flux losses to the chromosphere: about $8.6 \%$ of the total Alfvén wave energy flux (deposited between 1 and $52.5 R_{\mathrm{S}}$ ) flows back to the chromosphere, while only less than $2.8 \%$ of the Alfvén wave energy flux flows back to the chromosphere in 

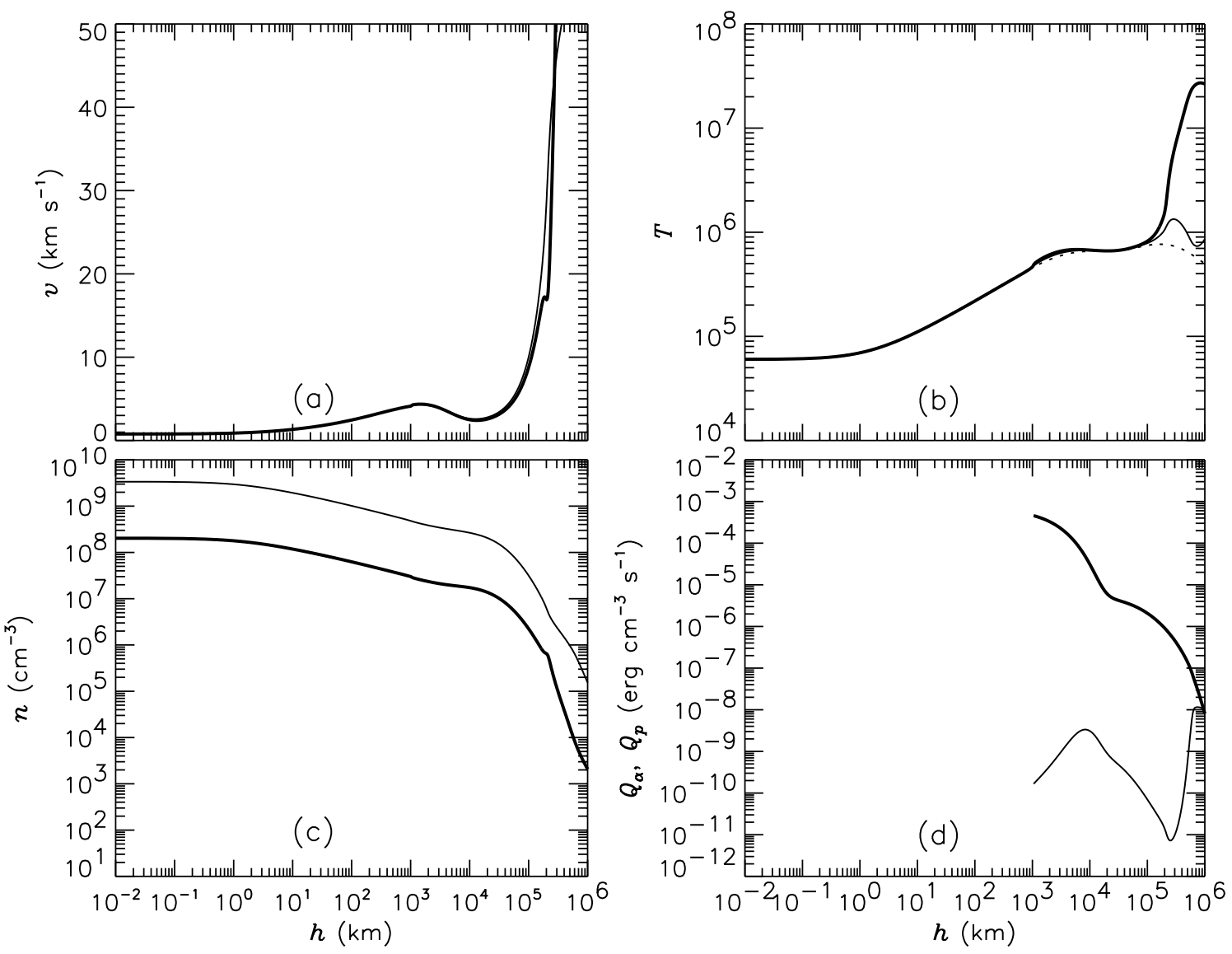

Fig. 10. A small region just above the lower boundary for Model 3. Here $f_{\mathrm{d}}=15000 \mathrm{~Hz}$.

Model 2. Hence a smaller $f_{\mathrm{d}}$ means more energy flux will be available for the solar wind acceleration, and a smaller particle flux and a faster wind may result (Leer \& Holzer 1980). Note, the height of the transition region changes more dramatically as $f_{\mathrm{d}}$ changes.

\subsection{Weak magnetic flux concentration}

Finally we briefly present Model 3 with a much weaker magnetic flux concentration in the supergranule convection zone boundaries. In Model 3, $f_{\mathrm{m} 1}=3.8$ and the magnetic field at the lower boundary is $B_{0}=36.1 \mathrm{G}$, so the magnetic field in interplanetary space is unchanged. Again the overall large scale velocity and temperature profiles (Model 3) are very close to Models 1 and 2 except a small region above the lower boundary, so only the region above the lower boundary is shown in Fig. 10. Because at the base of the corona, $\mathrm{d} f_{\mathrm{H}} / \mathrm{d} r$ is much smaller than in model 1 , and there is less wave energy available for alpha particles below 1.1 solar radii $\left(h<10^{5} \mathrm{~km}\right)$. This leads to a much colder corona below 1.1 solar radii and a less steep transition region (The maximum electron temperature is $7.67 \times 10^{5} \mathrm{~K}$.). Once again, species are in thermal equilibrium in the transition region $\left(h<10^{5} \mathrm{~km}\right)$. One noticeable feature of this model is that the flow speed at $T_{\mathrm{e}} \sim 10^{5} \mathrm{~K}$ level is quite small (less than $5 \mathrm{~km} \mathrm{~s}^{-1}$ ). The transition region is wide: the electron temperature increases from $6 \times 10^{4} \mathrm{~K}$ to $6 \times 10^{5} \mathrm{~K}$ in a distance of $3300 \mathrm{~km}$.
The model also produces reasonable proton particle flux: $2.07 \times 10^{8} \mathrm{~cm}^{-2} \mathrm{~s}^{-1}$. This model actually produces a faster wind than in Fig. 2. This can be understood since less wave energy is released just above the transition region, and the electron heat flux down to the region $T_{\mathrm{e}}<6 \times 10^{4} \mathrm{~K}$ is also smaller. Hence more wave energy flux becomes available for the solar wind acceleration. There is less energy at high frequencies suggesting less heat will be deposited in the subsonic region, leading to a faster but diluter wind (Leer \& Holzer 1980). It is found that if $f_{\mathrm{m} 1}$ is further decreased, with the same Alfvén wave amplitude, a steady state solution of a corona and wind can not be obtained.

\section{Discussion and concluding remarks}

Plasma heating in coronal funnels along open magnetic field lines by parallel propagating ion cyclotron waves is investigated in a three-fluid approach. It is shown that the Coulomb coupling between alpha particles and protons/electrons is strong enough to produce a sharp transition region and a hot corona by heating alpha particles alone. As shown by previous studies (Marsch \& Tu 1997a,b; Tu \& Marsch 1997; Hackeberg et al. 2000; Li 2002), a spectrum of ion cyclotron waves of wave spectrum index -1 is able to produce a transition region, a hot corona and to drive a fast solar wind if the wave spectrum extends to the ion cyclotron frequency range and the magnetic flux concentration in the super-granule convection zone boundaries is sufficiently strong. It is shown for the first time that by 
heating alpha particles alone, a sharp transition region, a hot corona and basically a fast solar wind can be produced. Hence, heavy ions may play a crucial role in the corona heating and the solar wind acceleration ( $\mathrm{Li}$ et al. 1997).

It is found that the highest frequency of the waves, or the location of the heating determines whether a thermal equilibrium can be established between minor ions and protons. As the maximum frequency is increased, alpha particles and protons end up farther away from thermal equilibrium. As the maximum frequency becomes smaller, alpha particles and protons are more likely to be in thermal equilibrium. However, if the maximum frequency is below a critical value, ion cyclotron resonance occurs far away from the transition region and a hot corona cannot be produced. The fact that heating alpha particles alone can produce a hot corona and wind has interesting implications on the theory of coronal heating and expansion. This study suggests that a corona can be created by solely heating minor ions. Of course, the coronal heating physics may be far more complicated than this study shows. Since there are many ions heavier than the alpha particles in the transition region and their gyro-frequencies are smaller than the gyro-frequency of alpha particles, those heavy ions will be heated first if parallel propagating ion cyclotron waves indeed are responsible for the coronal heating. Since the abundances of those minor ions are so small in the transition region and corona, it remains an open question whether the Coulomb coupling is able to efficiently transfer the energy from these minor ions to the major species (protons and electrons) (see Cranmer 2000; Hollweg \& Isenberg 2002).

For simplicity, fixed velocity distributions and spectra in the dissipation range are assumed. Tu \& Marsch (2001) have pointed out that due to cyclotron resonance, it is impossible for the spectrum to maintain a rigid shape. Ion cyclotron resonance may make the wave spectrum to have a cutoff at higher wave-numbers (Li et al. 2001). Physically, a sufficiently high spectral index shall mimic this effect. Since we have already taken a quite large spectral index -5 in the dissipation range, a larger spectral index is not going to change model results presented in this study. The energy distribution between protons and alpha particles is mainly determined by the dispersion relation. The model results are not sensitive to the dissipation range spectral index in this study. However the assumption of a fixed spectrum and velocity distribution may give unrealistic energy distributions between species. To tackle the wave heating problem in the corona, self-consistent spectral evolution including wave dispersion and kinetic treatment of particles are needed. The issue is currently under study by many authors (Tam \& Chang 1999; Galinsky \& Shevchenko 2000; Isenberg et al. 2000, 2001; Vocks \& Marsch 2001, 2002).

This study may have implications on the solar wind charge state investigations. By assuming plasma species in thermal equilibrium, coronal electron temperature can be calculated using charge fraction information from in situ charge state measurements. However, those calculations found that the coronal electron temperature above coronal holes is much higher than the electron temperature observed by SUMER (see, Ko et al. 1997; Esser et al. 1998). Since this investigation suggests that ions in the transition region may be already out of thermal equilibrium and have different velocities, the contradiction between SUMER and charge state calculations may be due to the loss of equilibrium in the transition region and corona. Recently, Esser \& Edgar (2001) found that the velocity difference can significantly reduce the discrepancy between observed in situ ion fractions and low coronal temperatures.

We have neglected the temperature anisotropy of species in this study and have adopted a collision dominated standard transport equations and modified classical thermal conduction for the three-fluid modelling. One has to recognize that it is still an open question to what degree the treatment is valid. Certainly Coulomb collisions are playing a major role in the transition region. As the plasma density deceases rapidly with an increasing height, collisions become less important in the inner corona. During the transition from collision dominated regime to collisionless regime, a refined description of species heat flux is needed. The heat flux of ions may also play a role in shaping the temperature anisotropy of the ions (Olsen et al. 1998; Olsen \& Leer 1999; Li 1999; Lie-Svendsen et al. 2001). A complete treatment to include species temperature anisotropies using high moment transport equations is under way.

Acknowledgements. This work is supported by PPARC rolling grant to University of Wales at Aberystwyth. Part of the work was supported by the grant NAG5-10873 to Smithsonian Astrophysical Observatory (SAO).

\section{References}

Axford, W. I., \& McKenzie, J. F. 1995, Solar wind 8, ed. D. Winterhalter, J. T. Gosling, S. R. Habbal, W. S. Kurth, \& M. Neugebauer, AIP, Dana Point, CA, p. 31

Axford, W. I., McKenzie, J. F., et al. 1999, Space Sci. Rev., 87, 25

Bavassano, B., Dobrowolny, M., Mariani, F., \& Ness, N. F. 1982, J. Geophys. Res., 87, 3617

Belcher, J. W., \& Davis, L., Jr. 1971, J. Geophys. Res., 76, 3534

Braginskii, S. I. 1965, in Reviews of Plasma Physics, 1, 204

Cranmer, S. R. 2000, ApJ, 532, 1197

Cranmer, S. R., Kohl, J. L., Noci, G., et al. 1999a, ApJ, 511, 481

Dere, K. P., Bartoe, J.-D. F., Brueckner, G. E., \& Recely, F., ApJ, 345, L95

Dowdy, J. F., Robin, D., \& Moore, R. L. 1986, Sol. Phys., 105, 35

Dusenberg, P. B., \& Hollweg, J. V. 1981, J. Geophys. Res., 86, 153

Esser, R., Edgar, R. J., \& Brickhouse, N. S. 1998, ApJ, 498, 448

Esser, R., Fineschi, S., Dobrzycka, D., et al. 1999, ApJ, 510, L63

Esser, R., \& Edgar, R. J. 2001, ApJ, 563, 1055

Gabriel, A. H. 1976, Phil. Trans. R. Roc. Lond. A., 281, 339

Hackenberg, P., Mann, P., \& Marsch, E. 2000, A\&A, 360, 1139

Hansteen, V. H., \& Leer, E. 1995, J. Geophys. Res., 100, 21, 577

Hansteen, V. H., Leer, E., \& Holzer, T. E. 1997, ApJ, 482, 498

Hassler, D. M., Dammasch, I. E., Lemaire, P., et al. 1999, Science, 283,810

Hollweg, J. V. 1986, J. Geophys. Res., 91, 4111,

Hollweg, J. V., \& Johnson, W. 1988, J. Geophys. Res., 93, 9547

Hollweg, J. V., \& Isenberg, P. A. 2002, J. Geophys. Res., 107(A7), 1147

Horbury, T. A., Balogh, A., Forsyth, R. J., \& Smith, E. J. 1995, Ann. Geophys., 13, 105

Hu, Y. Q., \& Habbal, S. R. 1999, J. Geophys. Res., 104, 17045

Hu, Y. Q., Esser, R., \& Habbal, S. R. 1997, J. Geophys. Res., 102, 14, 661 
Hu, Y. Q., Habbal, S. R., \& Li, X. 1999, J. Geophys. Res., 104, 24819

Hu, Y. Q., Esser, R., \& Habbal, S. R. 2000, J. Geophys. Res., 105, 2093

Isenberg, P. A. 1990, J. Geophys. Res., 95, 6437

Isenberg, P. A., \& Hollweg, J. V. 1982, J. Geophys. Res., 87, 5023

Isenberg, P. A., \& Hollweg, J. V. 1983, J. Geophys. Res., 88, 3923

Isenberg, P. A., Lee, M. A., \& Hollweg, J. V. 2000, Sol. Phys., 193, 247

Isenberg, P. A., Lee, M. A., \& Hollweg, J. V. 2001, J. Geophys. Res., 106,5649

Kohl, J. L., Noci, G., Antonucci, E., et al. 1998, ApJ, 501, L127

Kopp, R. A., \& Holzer, T. E. 1976, Sol. Phys., 49, 43

Leer, E., \& Holzer, T. E. 1980, J. Geophys. Res., 85, 4681

Li, X. 1999, J. Geophys. Res., 104, 19, 773

Li, X. 2002, ApJ, 571, L67

Li, X., \& Habbal, S. R. 1999, Sol. Phys., 190, 485

Li, X., Habbal, S. R., \& Hu, Y. Q. 1997, J. Geophys. Res., 102, 17, 419

Li, X., Habbal, S. R., Kohl, J., \& Noci, G. 1998, ApJ, 401, 133

Li, X., Habbal, S. R., Hollweg, J. V., \& Esser, R. 1999, J. Geophys. Res., 104, 2521

Li, H., Gary, P. S., \& Stawicki, O. 2001, Geophys. Res. Lett., 28, 1347

Lie-Svendsen, O., Leer, E., \& Hansteen, V. H. 2001, J. Geophys. Res., 106,8217

Lie-Svendsen, O., Hansteen, V. H., \& Leer, E. 2002, ApJ,

Marsch, E. 1999, Space Sc. Rev., 87, 1

Marsch, E., \& Tu, C. Y. 1990, J. Geophys. Res., 95, 8211

Marsch, E., \& Tu, C. Y. 1997a, Sol. Phys., 176, 87

Marsch, E., \& Tu, C. Y. 1997b, A\&A, 319, L17
Marsch, E., Goertz, C. K., \& Richter, A. K. 1982, J. Grophys. Res., 87,5030

McKenzie, J. F., Banaszkiewicz, M., \& Axford, W. I. 1995, A\&A, 303, 45

McKenzie, J. F., Axford, W. I., \& Banaszkiewicz, M. 1997, Geophys. Res. Lett., 24, 2877

Olsen, E. L., Leer, E., \& Lie-Svendsen, O. 1998, A\&A, 338, 747

Olsen, E. L., \& Leer, E. 1999, J. Geophys. Res., 104, 9963

Phillips, J. L., Bame, S. J., Feldman, W. C., et al. 1995, Science, 268, 1030

Rosner, R., Tucker, W. H., \& Vaiana, G. S. 1978, ApJ, 503, 475

Smith, E. J., Balogh, A., Neugebauer, M., \& McComas, D. 1995, Geophys. Res. Lett., 22, 3381

Spitzer, L., Jr. 1962, Physcis of Fully Ionized Gases (New York: Interscience)

Tam, S. W. Y., \& Chang, T. 1999, Geophys. Res. Lett., 26, 3189

Tu, C.-Y. 1987, Sol. Phys., 109, 149

Tu, C.-Y. 1988, J. Geophys. Res., 93, 7

Tu, C.-Y., Pu, Z. Y., \& Wei, F. S. 1984, J. Geophys. Res., 94, 11, 739

Tu, C.-Y., \& Marsch, E. 1997, Sol. Phys., 171, 363

Tu, C.-Y., Marsch, E., Wilhelm, K., \& Curdt, W. 1998, ApJ, 503, 475

Tu, C.-Y., \& Marsch, E. 2001, J. Geophys. Res., 106, 8233

Vocks, C., \& Marsch, E. 2001, Geophys. Res. Lett., 28, 1917

Vocks, C., \& Marsch, E. 2002, ApJ, 568, 1030

Warren, H. P., \& Hassler, D. M. 1999, J. Geophys. Res., 104, 9781

Wilhelm, K., Marsch, E., Dwivedi, B. N., et al. 1998, ApJ, 500, 1023

Wilhelm, K., Dammasch, I. E., Marsch, E., \& Hassler, D. M. 2000, A\&A, 353, 749 